
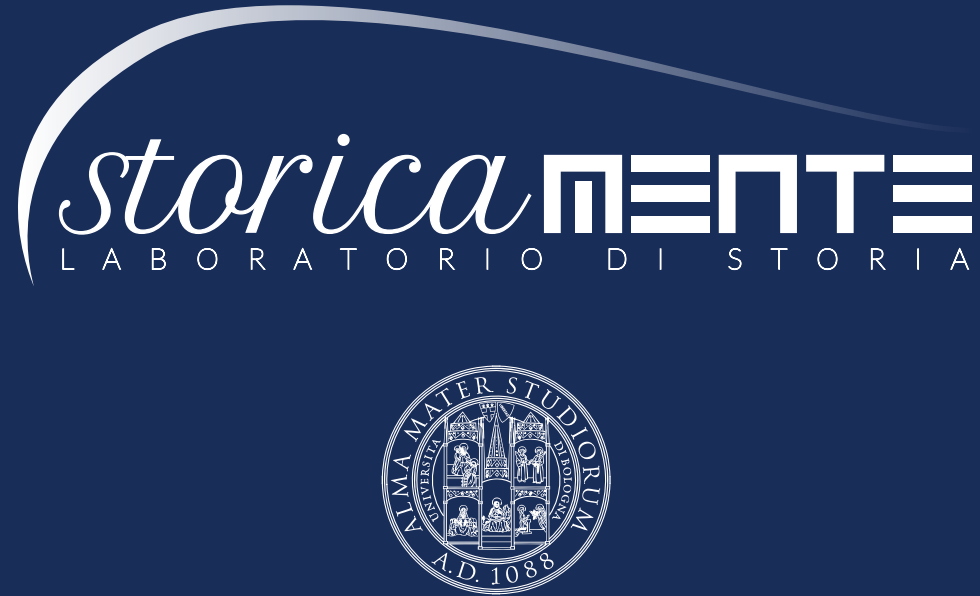

ALMA MATER STUDIORUM

Università di Bologna

Dipartimento di Storia Culture Civiltà

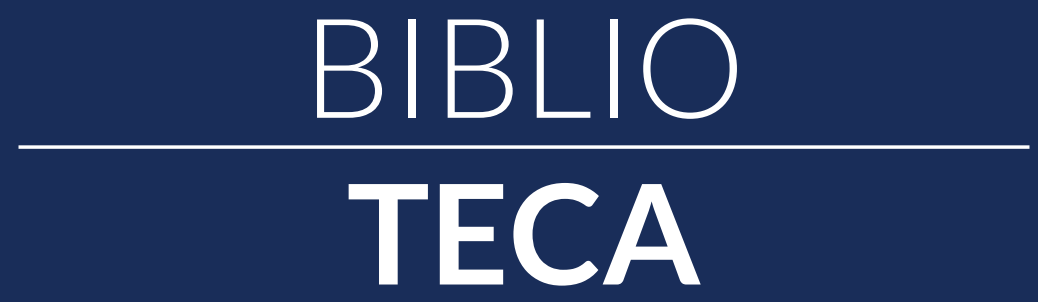

VIELLA 


\section{STORICAMENTE.ORG}

\section{Laboratorio di Storia}

Patrizia Gabrielli

Valeria Palumbo, "Non per me sola"

(C) Patrizia Gabrielli

Creative Commons BY-NC-ND 4.0

International License 2004- 2021

Volume: 2021

Issue: 17

Article number 4

Section: Biblioteca

Pages. 1-4

DOI: $10.52056 / 9788833138732 / 04$

ISSN: $1825-411 \mathrm{X}$

Publisher: Viella

Double blind peer review: Yes

Document type: Review

Research Areas: History; Literature; Women Studies

Published: 22/11/2021

Corresponding Address: Patrizia Gabrielli, Univ. di Siena, Dipartimento di scienze della formazione, scienze umane e della comunicazione interculturale, Campus del Pionta, viale Luigi Cittadini, 33, 52100, Arezzo, Italy 


\title{
Valeria Palumbo, "Non per me sola"
}

\author{
Patrizia Gabrielli
}

Univ. di Siena, Dipartimento di scienze della formazione, scienze umane e della comunicazione interculturale

patrizia.gabrielli@unisi.it

Valeria Palumbo, Non per me sola. Storia delle italiane attraverso i romanzi. Bari-Roma: Laterza, 2020. 230 pp.

Pagine e pagine di romanzi e racconti di illustri e apprezzati scrittori hanno delineato storie, tratteggiato profili di protagoniste affermate non di rado quali vere e proprie icone, «figure atemporali, come se non fossero frutto di un preciso momento storico e di una particolare cultura» (VII): si pensi anche solo all'insoddisfatta Madame Bovary o alla tragica Anna Karenina. La tradizione letteraria presenta una schiera di eroine coinvolte in trame e in scenari molteplici, dotate di personalità sfaccettate, dunque differenti tra loro, ma tutte frutto della fantasia e della creatività della penna di geni della letteratura come di mediocri scrittori che propongono figure stereotipate, presenza e al contempo alimento di un immaginario maschile.

Del fenomeno era ben consapevole Jolanda, pseudonimo adottato dalla marchesa Maria Majocchi Plattis, giornalista, direttrice, per alcuni anni, della nota e fortunata Cordelia, una delle prime riviste rivolte alle "signorine", autrice di romanzi amati dalle giovani le quali, alle soglie del nuovo secolo, seppure in bilico tra novità e conservazione, cominciano a manifestare un certo disagio e una certa insofferenza verso le strette maglie in cui versa la quotidianità della loro esistenza:

Voi ci volete a vostra immagine e somiglianza, avete la bontà di occuparvi de nostro miglioramento intellettuale e sociale, vi degnate di farci muovere con più o meno garbo nei vostri romanzi in cui si trovano persino donne ideali che conversano in latino... poi se una di noi, poveretta, un bel giorno trovandosi con tre idee in testa preferisce 
sedersi alla scrivania e metterle giù nella pace onesta della sua casa invece di oziare passeggiando o di far della maldicenza nei five o'clock thea, le gridate la croce addosso e la mandate a far la calza che qualche ora innanzi le toglieste di mano per farla assistere ad una conferenza dedicata a lei magari sull'origine dei Comuni e delle Monarchie (43).

Le considerazioni di Jolanda erano condivise da tante altre scrittrici le quali, con diversi stili, attraverso nuove eroine dotate di desideri, ambizioni, modi di sentire altri, hanno dato voce a altre dimensioni esistenziali, ad altri modelli: «le scrittrici, narrando il mondo, hanno rovesciato lo sguardo e il racconto» (201). Le donne creano nuove trame. Non sono più solo le artefici di quelle segnate sulla tela con il ricamo, considerato tradizionalmente la più nobile delle arti femminili, a partire dall'Ottocento sono molte a volerle scolpire con l'inchiostro sulla carta, scelta cui è sottesa la volontà di emergere dall'anonimato: con la scrittura esse affermano la propria soggettività nello scenario pubblico. Sulla scorta di una sicura padronanza della produzione letteraria di autrici più o meno note, l'A. consapevolmente sceglie di privilegiare le loro voci per dimostrare la presenza, a lungo rimasta nell'ombra, di «un'altra storia: delle donne stesse e dell'Italia» (VIII).

Dalle pagine di Grazia Deledda, Ada Negri, Sibilla Aleramo, Elsa Morante, Gianna Manzini, Alba De Cespedes, Melania G. Mazzucco, per citare solo alcune delle più note, come di Clotilde Scanabissi, Maria Messina, Annie Vivanti, meno conosciuti al grande pubblico, si snoda una narrazione, tematica e diacronica, che incrocia due secoli di storia della letteratura. Composto di tredici capitoli, introduzione e conclusioni, il volume attraversa varie questioni, tutte significative per la storia individuale e collettiva delle donne: dal rapporto con il padre all'amore, compreso quello lesbico per molti versi ritenuto persino innominabile, dal lavoro alla maternità, dal matrimonio all'adulterio, dalla moda ai viaggi, o meglio all'«impossibilità di muoversi in libertà» (44). L'articolata trattazione conferma largamente la tesi di Valeria Palumbo circa l'impegno di molte autrici nella riformulazione del canone letterario. Esse hanno denunciato sfruttamento e solitudine, povertà, sacrifici, limiti, costrizioni morali e, con maggiore o minore forza, hanno lasciato intravvedere alle donne possibilità di emancipazione: «in sintesi, la letteratura femminile ci ha mostrato che è possibile approdare a un lieto 
fine anche per le donne che rifiutano gli stretti binari della "normalità" e della "rispettabilità" borghese» (204). Esse operano una decostruzione del modello femminile codificato, un'operazione dalla quale, data la reciprocità dei modelli di genere, investe le pratiche discorsive sul maschile. Gli uomini tratteggiati dalla letteratura femminile si discostano, almeno in parte, dall'immagine virile più consolidata.

Sebbene, come accennato, l'A. consapevolmente scelga quale fonte primaria la scrittura letteraria, merita sottolineare che, al fine di inquadrare usi, comportamenti, modelli il volume propone molti richiami a codici e leggi, alla stampa. Esaminando norme sociali, consuetudini, amori, sentimenti, passioni presenti nell'universo immaginifico di numerose scrittrici, l'A. ridefinisce spazi privati e pubblici e si sofferma su alcuni momenti e grandi eventi storici. A tale proposito si possono citare i riferimenti al dibattito, compreso quello femminista, sulla condizione delle lavoratrici. Se si volge attenzione alla storia o meglio alla sua ri-scrittura, merita menzione il capitolo dedicato alla guerra e alla Resistenza nel quale Palumbo, attingendo ad alcuni romanzi, è il caso di Renata Viganò, infrange le più consuete rappresentazioni sulla Resistenza e si sofferma sulla violenza e sullo stupro. Citando Ida - l'eroina «sempre calata nel mondo quotidiano» (153), a differenza degli eroi classici che vivono e operano nel mondo dell'eccellenza - la protagonista di una delle più apprezzate opere di Elsa Morante, La storia, Valeria Palumbo sottolinea le differenze profonde che connotano questo personaggio e Rosetta, la figlia di Cesira, entrambe protagoniste de La ciociara, di Alberto Moravia. Più di un approfondimento in tal senso viene dall'analisi delle pratiche discorsive che nel secondo dopoguerra ruotano intorno allo stupro. Così, richiamando l'intervento di Maria Maddalena Rossi in Parlamento, l'A. non dimentica la santificazione di Maria Goretti nel 1950: «è interessante anche notare che Maria diventò santa quando il problema della povertà nell'Agro Pontino era stato, almeno temporaneamente, risolto e quindi il tema della "moralità" delle donne, come sempre corrotte dalla modernità e dal benessere, diventava più cogente per la Chiesa» (156). La rappresentazione della santa è un punto privilegiato per sottolineare diversi immaginari sullo stupro: «nella vicenda di Maria Goretti avviene l'esatto contrario di quanto fa Ida in La Storia. Ida subisce la violenza del soldato tedesco, più stupita che umiliata. E poi continua a vivere. Come hanno fatto le donne vittime degli stupri della Ciociaria» (157). 
La ricchezza delle voci e delle tematiche affrontate, la scrittura fluida accompagnano la lettura di queste pagine, ma a coinvolgere lettrici e lettori è anche la consapevolezza manifestata dall'A. sulle tematiche inerenti la parità di genere. Un coinvolgimento, direi posizionamento, che non inficia in alcun modo analisi e interpretazione, aggiunge invece valore a una ricerca che non elude le tante domande del presente sui modelli di genere e sulla loro trasmissione alle nuove generazioni. Interrogativi difficili, alla cui soluzione - come con sensibilità suggerisce questo libro - può dare un contributo la letteratura: «la narrativa può ancora fare la sua parte: sia per l'inerzia di certi vecchi schemi (valgono per la femminilità come per la virilità), sia perché è importante dare alle più giovani la certezza che non avanzano allo scoperto, e che dietro di loro ci sono una lunga elaborazione e una lunga storia di emancipazione e di conquiste» (205). 